\title{
Das Konzept der «Co-benefits» von Gesundheit und Umwelt
}

\section{Julia Gonzalez Holguera ${ }^{a}$ und Nicolas Senn ${ }^{b}$}

a Interdisziplinäres Zentrum für Nachhaltigkeit, Universität Lausanne; b Abteilung Familienmedizin, Unisanté, Universität Lausanne

Der menschengemachte Klimawandel gefährdet die in der Medizin erzielten Fortschritte. Die Schweizerische Akademie der Medizinischen Wissenschaften (SAMW) beantwortet im Rahmen ihres 2019 erschienenen Positionspapiers zur nachhaltigen Entwicklung des Gesundheitssystems Fragen zwischen Gesundheit und Umwelt (Konzept der "Co-benefits») und unterstützt eine gemeinsam mit der Revue Médicale Suisse veröffentlichte Literaturübersicht zum Thema.

Die Auswirkungen von Umweltschäden auf die Gesundheit des Menschen werden immer offensichtlicher und bedeuten eine grosse Herausforderung für die öffentliche Gesundheit auf der ganzen Welt. In den letzten Jahrzehnten konnten dank Gesundheitsförderung und medizinischen Errungenschaften die Lebenserwartung erhöht und der Gesundheitszustand der Allgemeinbevölkerung verbessert werden. Diese Fortschritte sind aber durch die menschengemachte Umweltzerstörung wieder in Gefahr. Die Covid-19-Pandemie veranschaulicht mit ihren verheerenden gesellschaftlichen und gesundheitlichen Folgen eindrücklich die seit Jahrzehn- ten geäusserten Befürchtungen einer Zunahme von Zoonosen, die insbesondere auf die Zerstörung natürlicher Lebensräume zurückzuführen sind [1, 2].

Immer mehr Autorinnen und Autoren von Leitartikeln und medizinische Fachverbände weisen darauf hin, welche Rolle Gesundheitsfachleute beim Übergang hin $\mathrm{zu}$ einer ökologischeren Lebensweise spielen sollten [3-5]. Die Zahl der Studien, welche sich damit befassen, wie diese Bestrebungen umgesetzt werden können, wie effektiv sie sind und wie die grundlegenden, engen Verbindungen zwischen Gesundheit und Umwelt aussehen, ist momentan eher klein.

\section{Für Mensch und Umwelt vorteilhafte Massnahme}
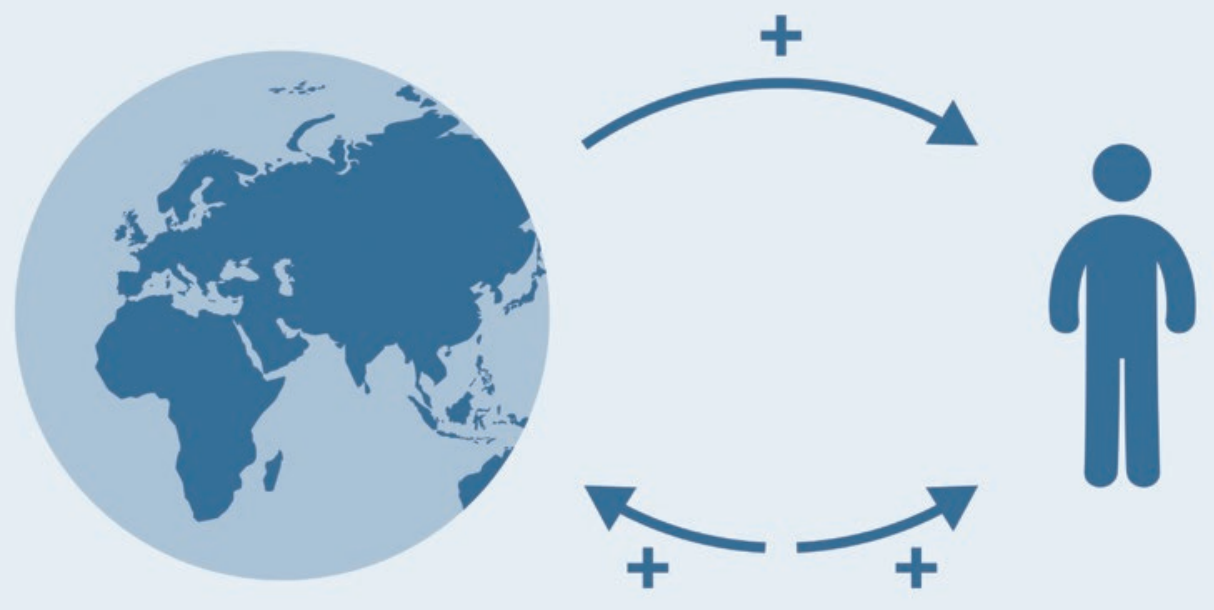

Abbildung 1: Schematische Darstellung der Co-benefits von Gesundheit und Umwelt (Quelle: SAMW). 


\section{Für mehr Nachhaltigkeit}

Anknüpfend an einen im SAMW Bulletin 4/2020 erschienenen Schwerpunkt [6] präsentiert nun eine Literaturübersicht über die positiven Wechselwirkungen zwischen Umwelt und Gesundheit den aktuellen Wissensstand für medizinsche Fachpersonen. Die Literaturübersicht soll die Entwicklung unserer Gesellschaft zu mehr Nachhaltigkeit über das Konzept der «Co-benefits» zwischen Gesundheit und Umwelt begleiten. Die Übersicht (www.revmed.ch/cobenefices) wurde von der Abteilung für Familienmedizin der Unisanté und vom Interdisziplinären Zentrum für Nachhaltigkeit der Universität Lausanne zusammengestellt, von der Revue Médicale Suisse (RMS) auf Französisch veröffentlicht und kürzlich in Zusammenarbeit mit der SAMW unter dem Titel «Health and Environment Co-Benefits: Concepts and Recommendations for Clinical Practice» ins Englische übersetzt. Warum wurde diese Literaturübersicht publiziert? Müll trennen allein genügt in der Tat nicht. Es braucht einen echten Paradigmenwechsel, sowohl in unserer Gesellschaft als auch im Gesundheitssystem und auch bezüglich unserer Auffassung von Gesundheit. In der Literaturübersicht werden einige für diese Überlegungen wichtige Faktoren herausgegriffen.

\section{Konzept der «Co-benefits»}

Der Begriff «Co-benefits» beschreibt die Auswirkungen von Massnahmen zur Verringerung von Umweltschäden auf die menschliche Gesundheit oder umgekehrt von Gesundheitsförderungsmassnahmen auf den Schutz der Umwelt (siehe Abb. 1). Solche Massnahmen werden eher akzeptiert, wenn auf ihre lokalen und unmittelbaren Vorteile hingewiesen wird [7]. Konkret kann dies bedeuten, dass Ärztinnen und Ärzte be-

\section{Studien zeigen, dass es Ärzten unangenehm} ist, mit ihren Patienten über die Umwelt zu sprechen, weil es ihnen an Wissen fehlt.

stimmte Themen wie aktive Mobilität, gesunde Ernährung und mehr Kontakt mit der Natur gegenüber den Patientinnen und Patienten direkt ansprechen und mit ihnen Entscheidungen in Energiefragen und gemeinschaftlichem Engagement gemäss den Empfehlungen der WONCA (Weltorganisation für Allgemeinund Familienmedizin) diskutieren [8]. Das Konzept der "Co-benefits» mag für viele selbstverständlich sein. Studien zeigen jedoch, dass sich viele Ärztinnen und Ärzte schwertun, mit ihren Patientinnen und Patienten über Umweltthemen zu sprechen, weil es ihnen an
Kenntnissen und an einem adäquaten konzeptuellen Gesundheitsbegriff fehlt, der zum Ausdruck bringt, dass die Gesundheit des Menschen eng mit der Gesundheit der natürlichen Ökosysteme verknüpft ist [9]. Eine noch laufende Studie zeigt, dass viele Ärztinnen und Ärzte mit dem Begriff «Co-benefits» wenig vertraut sind (André, et al., Studie läuft noch). In der klinischen Praxis kann dieses Konzept genutzt werden, um Einzelne zu Verhaltensänderungen zu bewegen und somit einen doppelten Vorteil zu erzielen: Verbesse-

Das Gesundheitspersonal spielt eine wichtige Rolle in der Unterstützung seiner Patienten bei der Änderung ihrer Essgewohnheiten.

rung der Gesundheit jedes einzelnen Menschen und damit der gesamten Bevölkerung sowie Verringerung der Umweltschäden. Zusammen mit entsprechenden strukturellen Massnahmen bietet das Konzept folglich eine innovative Perspektive, um Fragen der ökologischen Nachhaltigkeit in der klinischen Praxis fächerübergreifend zu behandeln.

In Pflegeeinrichtungen können unterschiedliche Strategien genutzt werden, um den $\mathrm{CO}_{2}$-Abdruck zu verkleinern. $\mathrm{Zu}$ den wichtigen Massnahmen zählen: die Patientinnen, Patienten und das Personal ermutigen, zu Fuss oder mit dem Fahrrad zu kommen, die Arbeitsfläche pro Arzt verkleinern, eine umweltbewusste Verschreibungspraxis einführen oder die Benutzung steril verpackter Baumwollkompressen reduzieren. Solche Empfehlungen finden sich in Initiativen wie Green Hospital (www.greenhospital.ch) oder in der Ausgabe der Revue Médicale Suisse für Arztpraxen vom Mai 2021 [10].

\section{Nähere Betrachtung: Mobilität, Ernährung, Natur}

Die Literaturübersicht präsentiert das Konzept der «Co-benefits» anhand der drei Themen Ernährung, aktive Mobilität und Kontakt mit der Natur im Detail. Studien belegen, dass in diesen Bereichen mit gewissen strukturellen Umgestaltungen und individuellen Verhaltensänderungen die Umweltbelastung verringert und gleichzeitig direkte positive Nebeneffekte für die Gesundheit erzielt werden können.

Dass die Ernährung immer $\mathrm{CO}_{2}$-lastiger wird, ist ein globaler Trend. Insbesondere der Konsum tierischer Produkte nimmt stark zu. Basierend auf dem prognostizierten Wachstum der Weltbevölkerung von rund $30 \%$ (8,5 bis 10 Milliarden im Jahr 2050) und einem der erwarteten Entwicklung des Lebensstandards entsprechenden Ernährungstrend könnten die durch die 
Ernährung verursachten Treibhausgasemissionen bis 2050 um 80 bis $90 \%$ steigen. Der Bedarf an Landwirtschaftsflächen wird laut Prognosen um 67\%, der Trinkwasserverbrauch um 65\% und der Einsatz von Phosphor und Stickstoff um 54 bzw. 51\% zunehmen. Dies vor dem Hintergrund, dass die Landwirtschaft bereits heute ein wichtiger Faktor bei der Entstehung von Umweltschäden ist [11].

Das positive Gegenstück zu den Verhaltensänderungen (insbesondere der Konsumrückgang von stark verarbeiteten Lebensmitteln sowie von Fleisch) besteht in den Auswirkungen auf chronische Erkrankungen wie Fettleibigkeit und Diabetes, von denen weltweit mehrere Milliarden Menschen betroffen sind. Zahlreiche Studien weisen auf die Möglichkeit einer Entwicklung zu gesünderen und nachhaltigeren Ernährungsgewohnheiten hin. Gesundheitsfachleute spielen dabei eine wichtige Rolle: sie sollten die Bevölkerung bei der Änderung ihrer Ernährungsgewohnheiten hin zu mehr Getreide, Obst, Gemüse und Hülsenfrüchten anstelle von übermässigem Konsum von rotem Fleisch, Milchprodukten, Eiern und stark verarbeiteten Produkten begleiten [12].

\section{Rolle der medizinischen Fachkräfte}

Angesichts der Tatsache, dass die WHO für Erwachsene mindestens 150 Minuten körperliche Betätigung pro Woche empfiehlt, immer mehr Menschen dieses Ziel jedoch nicht erreichen, stellt sich die Frage: Inwieweit könnten medizinische Fachpersonen zu mehr körperlicher Aktivität ermutigen, um den zunehmenden gleichzeitig einen Beitrag zur Reduktion von Umweltund Lärmverschmutzung leisten? Könnten sie auch dabei helfen, die Biodiversität zu erhalten, indem sie auf die Vorteile von Gemeinschaftsgärten und städtischen Grünflächen hinweisen, die die körperliche Betätigung, eine bessere Stressbewältigung und eine mögliche Reduktion von Allergien fördern und das soziale Kapital erhöhen? In der Fachliteratur gibt es diverse Hinweise darauf, dass ein vermehrter Aufenthalt in der Natur zahlreiche gesundheitliche Vorteile mit sich bringt (Allergien, psychische Gesundheit, Sozialisation und allgemeine körperliche Fitness).

Die Berücksichtigung von Umweltperspektiven in der klinischen Praxis mag zwar für einige eine Selbstverständlichkeit sein, erfordert aber insgesamt ein Überdenken der Begriffe Gesundheit und Krankheit sowie der daraus abgeleiteten Gesundheitsdienstleistungen. Die von der SAMW und der RMS veröffentlichte Literaturübersicht über die "Co-benefits» zwischen Gesundheit und Umwelt versucht, entsprechende Denkanstösse zu geben.

Die Literaturübersicht ist unter der Adresse www.revmed.ch/ cobenefices auf Französisch und Englisch verfügbar.

\section{Literatur}

1 Daszak P. Pandemic Report: Escaping the 'Era of Pandemics'; IPBES Workshop Report on Biodiversity and Ecosystem Intergovernmental Platform on Biodiversity and Ecosystem Services. 2020.

2 McMahon BJ, Morand S, Gray JS. Ecosystem change and zoonoses in the Anthropocene. Zoonoses Public Health. 2018;65(7):755-65.

3 Bain PG, et al. Co-benefits of addressing climate change can motivate action around the world. Nature Climate Change. 2016;6(2): $154-7$.

4 Watts N, et al. The 2019 report of The Lancet Countdown on health and climate change: ensuring that the health of a child born today is not defined by a changing climate. The Lancet. 2019;394(10211): 1836-78.

5 WONCA, P.H.A. Clinicians for Planetary Health Working Group, Declaration calling for family doctors of the world to act on planetary health. 2019.

6 Senn N. Santé et environnement naturel: un lien fort. Bulletin SAMW/ASSM. 4;2020.

7 Karlsson M, Alfredsson E, Westling N. Climate policy co-benefits: a review. Climate Policy. 2020;20(3):292-316.

8 https://www.globalfamilydoctor.com/Resources/A-Ztopiclisting/ EnvironmentalMedicine.aspx

9 Boland TM, Temte JL. Family Medicine Patient and Physician Attitudes Toward Climate Change and Health in Wisconsin. Wilderness Environ Med. 2019.

10 Nicolet J, et al. Recommandations pour l'écoconception des cabinets de médecine de famille. Revue Médicale Suisse. Mai 2021;738.

11 Springmann $M$, et al. Options for keeping the food system within environmental limits. Nature. 2018;562(7728):519-25.

12 Willett W, et al. Food in the Anthropocene: the EAT-Lancet Commission on healthy diets from sustainable food systems. Lancet. 2019;393(10170):447-92 